\title{
Synopsis of warty leaf beetle genera of the World (Coleoptera, Chrysomelidae, Cryptocephalinae, Chlamisini)
}

\author{
M. Lourdes Chamorro-Lacayo', Alexander S. Konstantinov²
}

I Department of Entomology, Smithsonian University, P.O. Box 37012, MRC 187, National Museum of Natural History, Washington DC 20013-7012, U.S.A. 2 Systematic Entomology Laboratory, PSI, Agricultural Research Service, U.S. Department of Agriculture, clo Smithsonian Institution, P.O. Box 37012, National Museum of Natural History, MRC 168 Washington, DC 20013-7012, U.S.A.

Corresponding authors: M. Lourdes Chamorro-Lacayo (chamorrom@si.edu; lourdes.chamorro@gmail.com), AlexanderS. Konstantinov (alex.konstantinov@ars.usda.gov)

Academic editor: Terry Erwin | Received 26 January 2009 | Accepted 28 April 2009 | Published 30 April 2009

Citation: Chamorro-Lacayo ML, Konstantinov AS (2009) Synopsis of warty leaf beetle genera of the World (Coleoptera, Chrysomelidae, Cryptocephalinae, Chlamisini). ZooKeys 8: 63-88. doi: 10.3897/zookeys.8.90

\begin{abstract}
The 11 world genera of Chlamisini Gressitt are reviewed, diagnosed, and illustrated. A key for their identification is provided. A replacement name is proposed, Kakita Chamorro-Lacayo \& Konstantinov, nom. n., for Ceratochlamys Bokermann, 1961, a junior homonym of Ceratochlamys Habe, 1946 (Mollusca). Chlamisus rousei Medvedev, 1993 is designated as a junior synonym of Chlamisus straminea Suffrian, 1866 , syn. n.
\end{abstract}

\section{Keywords}

Chrysomelidae, Chlamisini, Cryptocephalinae, leaf beetles, key, genera, world, new name

\section{Introduction}

Chlamisini Gressitt constitute a relatively small tribe in the leaf beetle subfamily Cryptocephalinae Gyllenhal with approximately 500 species currently classified in 11 genera (Blackwelder 1946; Monrós 1952; Seeno and Wilcox 1982). The distribution of species in the tribe is largely circumtropical. The majority of species and 10 of the 11 genera occur in the Neotropical Region, particularly in the Amazon Basin (Table 1). The only genus not represented in the Neotropical region, Hymetes Lacordaire, 1848, is

Copyright ML Chamorro-Lacayo, AS Konstantinov. This is an open access article distributed under the terms of the Creative Commons Attribution License, which permits unrestricted use, distribution, and reproduction in any medium, provided the original author and source are credited. 
known to occur only in the Oriental region. Of the 10 genera found in the Neotropics, four are endemic. The largest genus, Chlamisus Rafinesque, has been reported from all biogeographic regions and it is the only chlamisine genus to be found in the Palearctic and Australasian regions.

Adult chlamisines are distinguished readily from other leaf beetles by their tuberculate or warty dorsal body surface (resembling caterpillar droppings), by their legs fitting into depressions, and by the presence of antennal grooves adjacent to the prosternal process into which the short serrate antennae fit. Hence, these beetles have the ability to tightly withdraw all appendages to form a compact cylinder and successfully mimic small flower buds, caterpillar droppings, or other forms of debris or excrement. Most are dark colored, but some are metallic or light with dark velvety spots.

Chlamisini, as well as other Cryptocephalines, have an interesting and unique life history. Each egg is individually covered by the mother, with a mixture of her own feces and rectal secretions to form a scatoshell, before releasing it into the environment (LeSage 1982, 1984; Erber 1988; Brown and Funk 2005). The individual plates that make up the scatoshell (egg-case) are thought to be unique to each species (Erber 1988). After eclosion, the larva retains this scatoshell. With each subsequent instar, the growing larva builds onto its inherited scatoshell with its own pliable feces (Brown and Funk 2005; Chaboo, Brown and Funk 2008; LeSage 1982, 1984). Finally, pupation takes place within their cases. These cases may not only afford larvae mechanical protection, but they may help to disguise them from predators by effectively resembling auxiliary plant buds.

The classification of Chlamisini remains largely unchanged from that proposed by Lacordaire (1848) more than 150 years ago, which has been properly criticized as being inadequate (Karren 1972). Chlamisus Rafinesque 1815 (=Chlamys Knoch,

Table I. List of World Chlamisini genera and their distributional ranges. NA = Nearctic; NT $=$ Neotropical; AT = Afrotropical; WP = West Palearctic; EP $=$ East Palearctic; $\mathrm{OR}=$ Oriental; $\mathrm{AU}=$ Australasian (Monrós 1952; Karren 1966, 1972; Reid 1991).

\begin{tabular}{|c|c|c|c|c|c|c|c|c|c|}
\hline Genus & Author & Year & NA & NT & AT & WP & EP & OR & $\mathrm{AU}$ \\
\hline Chlamisus & Rafinesque & 1815 & $\mathrm{X}$ & $\mathrm{X}$ & $\mathrm{X}$ & $\mathrm{X}$ & $\mathrm{X}$ & $\mathrm{X}$ & $\mathrm{X}$ \\
\hline Diplacaspis & Jacobson & 1924 & $\mathrm{X}$ & X & & & & & \\
\hline Exema & Lacordaire & 1848 & $\mathrm{X}$ & $\mathrm{X}$ & & & & $\mathrm{X}$ & \\
\hline Neochlamisus & Karren & 1972 & $\mathrm{X}$ & $\mathrm{X}$ & & & & & \\
\hline Pseudochlamys & Lacordaire & 1848 & $\mathrm{X}$ & $\mathrm{X}$ & & & & & \\
\hline Melittochlamys & Monrós & 1948 & & $\mathrm{X}$ & & & & & \\
\hline Aulacochlamys & Monrós & 1952 & & $\mathrm{X}$ & $\mathrm{X}$ & & & $\mathrm{X}$ & \\
\hline Fulcidax & Voet & 1806 & & $X$ & & & & & \\
\hline Hymetes & Lacordaire & 1848 & & & & & & $\mathrm{X}$ & \\
\hline Kakita & $\begin{array}{l}\text { Chamorro-L. \& } \\
\text { Konstantinov }\end{array}$ & 2009 & & $\mathrm{X}$ & & & & & \\
\hline Carcinobaena & Lacordaire & 1848 & & $\mathrm{X}$ & & & & & \\
\hline
\end{tabular}


1801, nec Bolten, 1798) is the type genus of the tribe Chlamisini Gressitt, 1946, which is based on the valid name of the original nominal type genus (i.e., Chlamys). Nonetheless, the invalid name Fulcidacinae, based on the genus Fulcidax Voet, 1806, has been used previously (Jacobson 1924; Navajas 1944a,b; Chûjô 1940, 1942; Braga et al. 1999). To date, Chlamisus includes the majority of species in the tribe and has become a catch-all genus in need of a comprehensive taxonomic revision (Karren 1972; Monrós 1952; Reid 1991). Lacordaire (1848) proposed 14 species groups in Chlamisus.

The second oldest name in the tribe is Fulcidax, a senior synonym of Poropleura Lacordaire, 1848. In addition to Poropleura, five additional genera were proposed by Lacordaire (1848): Carcinobaena Lacordaire, Diaspis Lacordaire (= Diplacaspis Jacobson, 1924), Exema Lacordaire, Hymetes Lacordaire, and Pseudochlamys Lacordaire. Monrós $(1948,1952)$ established 2 genera, Melittochlamys Monrós for the first 7 species included by Lacordaire (1848) in species group 1 of Chlamisus, and Aulacochlamys Monrós for several species in Exema. Bokermann (1961) proposed the genus Ceratochlamys Bokermann for an unusual species from Brazil; however, this name is a junior homonym of Ceratochlamys Habe, 1946 in Mollusca and a replacement name is here proposed. The most recent addition to the tribe, Neochlamisus Karren, was described almost 4 decades ago. The genus was established as a result of a comprehensive revision of North American Chlamisinae north of Mexico, and it consists of a number of species formerly included in Chlamisus and Diplacaspis (Karren 1972). Major chlamisine revisions have been regional, e.g., Monrós (1952) and Karren (1966, 1972) for Argentina and North America north of Mexico, respectively. These treatments comprise the most recent taxonomic activity in the tribe.

Motivation for this study came from the realization that identification tools for chlamisine genera, mainly for the Neotropical fauna, are outdated, incomplete, or difficult to use, particularly when dealing with very similar taxa (i.e., Chlamisus, Diplacaspis, Neochlamisus, Pseudochlamys, Fulcidax, and Exema). As with most Neotropical Chrysomelidae, identification at all taxonomic levels below tribal, presents a major challenge, and generic differentiation of chlamisines is no exception. Yet, no single key offers the tools to confidently segregate species into genera. Therefore, the objectives of this study were to provide comprehensive, accurate, and lucid identification tools to world chlamisine genera and to identify potential areas of research. This was achieved by studying the type species of each genus (when available) and several congeners, and by comparing traditional and novel characters across all taxa. The product consisted of a dichotomous key to all world genera, as well as analogous taxonomic diagnoses and high resolution images/illustrations for each genus.

Even though we follow the classification proposed by Lacordaire (1848) and our study is based on original generic concepts, we echo the sentiment expressed by Karren (1972) and Reid (1991) that there is a need for a complete reassessment of generic boundaries within Chlamisini, particularly Chlamisus, to reflect natural groups. This study is the first step towards a comprehensive revision of Chlamisini, which will center largely around taxa from the Neotropical Region. Characters identified in this study 
will provide a basis from which to address generic concepts in the future. Complete disarticulation of key taxa from each genus and morphological comparison among the genera was beyond the scope of this study.

\section{Materials and methods}

Our treatment of all chlamisine genera is based on examination of the type species of each genus, when available. Additional representatives of each genus were examined (see material examined section under each generic treatment). All material examined is housed in the entomology collection of the National Museum of Natural History, Smithsonian Institution (NMNH). This institution holds the Monrós collection as well as many chlamisines identified by Karren and Bokermann. Under each genus a list of the material examined is provided; it includes exact label data for each specimen [all labels are listed as they appear from top to bottom on the pin and each label starts with a lower case letter separated by a forward dash (/)]. Genera are listed in alphabetical order. In addition to observations made of available specimens, the dichotomous key takes into account characters used by Monrós (1952), Karren (1972), and Riley et al. (2002). Terminology for morphological structures follows Karren (1972), ChamorroLacayo and Konstantinov (2004), and Chamorro-Lacayo et al. (2006).

\section{Diagnoses of World Chlamisini genera}

\section{Aulacochlamys Monrós}

(Figs. 1 A, B; 2 A, B; 3 A; 4, A; 5 A)

Aulacochlamys Monrós, 1952: 657. Type species: Exema costicollis Lacordaire, 1848, by original designation. Seeno and Wilcox, 1982: 43 (catalog).

Diagnosis. Length $2.00-2.80 \mathrm{~mm}$, width $1.18-1.72 \mathrm{~mm}$. General body shape cylindrical. Antenna serrate beyond $5^{\text {th }}$ antennomere; $3^{\text {rd }}, 4^{\text {th }}$, and $5^{\text {th }}$ antennomeres slightly widened, but not dilated distally. Pronotum medially elevated, with six distinct, small, sharp, longitudinal carinae, which converge medially near posterior margin. Pronotal base opposite mesoscutellum (posterior pronotal lobe) with or without notch (Fig. 1A). Prosternum gradually narrowing posteriorly, broadening before apex. Mesoscutellum very short, transverse. Anterior margin of metasternum concave. Metascutellum concealed by elytra. Elytral suture completely serrate, although serration weak near scutellum. Elytral tubercules well developed. Tibiae slightly curved, cylindrical. Foreand midtibial apices without claw. Tarsal claw appendiculate.

Distribution. Pantropical, except Australia (Monrós, 1952).

Remarks. Aulacochlamys resembles Chlamisus Rafinesque in overall body shape, size and color; however, the six longitudinal carinae on the pronotum immediately dis- 


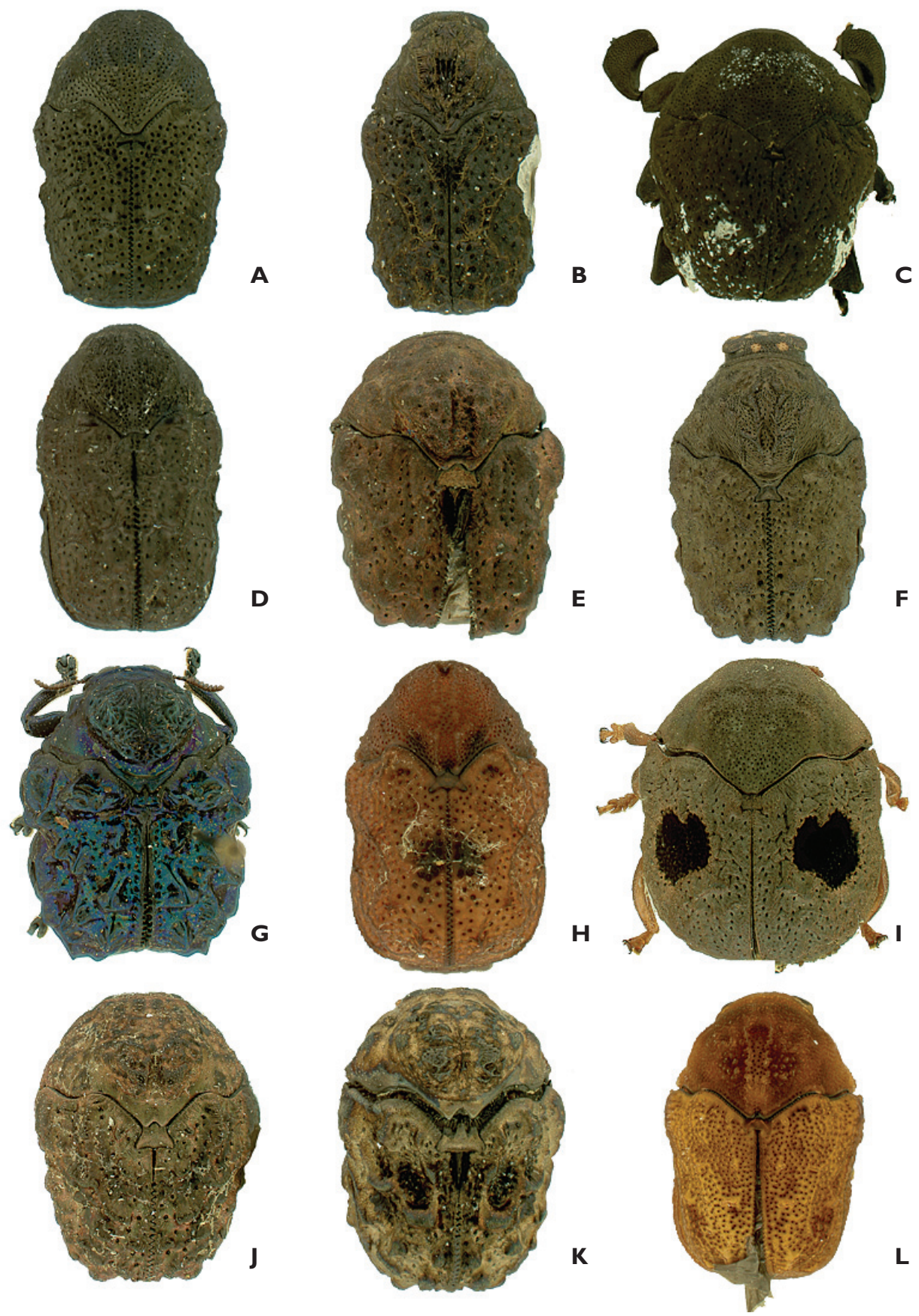

Figure I. Chlamisini, dorsal view. A, Aulacochlamys distincta (Achard). B, A. costicollis (Lacordaire. C, Carcinobaena pilula (Klug). D, Chlamisus foveolatus (Knoch). E, Diplacaspis prosternalis (Schaeffer). F, Exema elliptica Karren. G, Fulcidax coelestina (Lacordaire). H, Hymetes javana Lacordaire. I, Melittochlamys specula (Klug). J, Neochlamisus insularis (Schaeffer). K, N. velutinus Karren. L, Pseudochlamys megalostomoides Lacordaire $q$. 
tinguish it from Chlamisus. The absence of spines on the fore- and midtibiae also differentiates most studied species of Aulacochlamys from Chlamisus; however, this character may be sexually dimorphic in Chlamisus (Karren 1972).

A total of 32 species were included in this genus by Monrós (1952); 3 Afrotropical, 21 Neotropical, and 8 Oriental.

\section{Material examined.}

Aulacochlamys costicollis (Lacordaire):

1) a. [Brazil] Corumba, Matt. Grosso/ b. F. Monrós Collection, 1959/ c. Aulacochlamys costicollis (Lac.) F. Monrós det. 1950. 2) a. Bolivia, Nor. Ungas, Co-

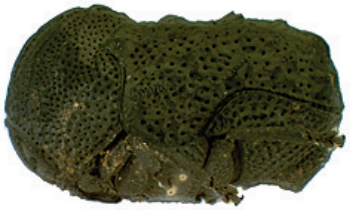

A

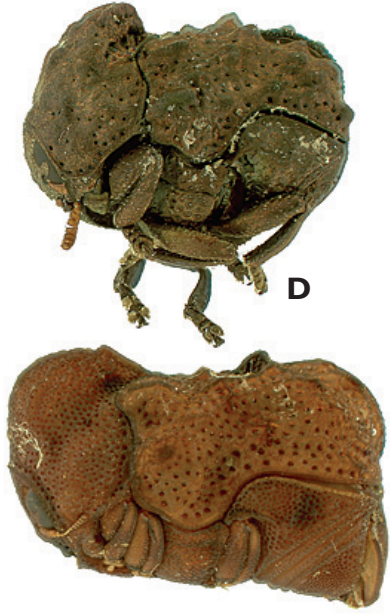

G

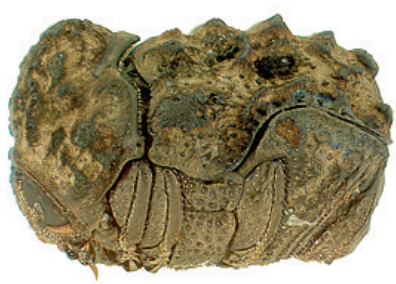

J

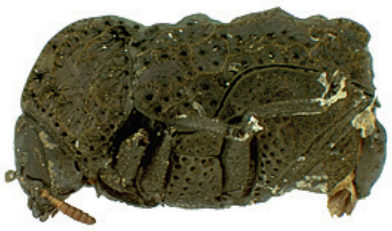

B
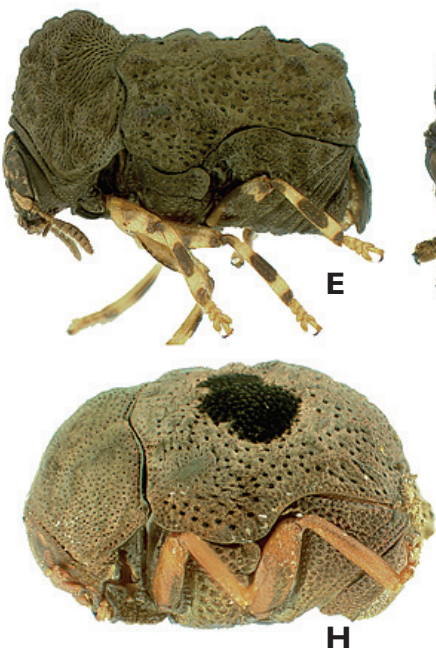

H

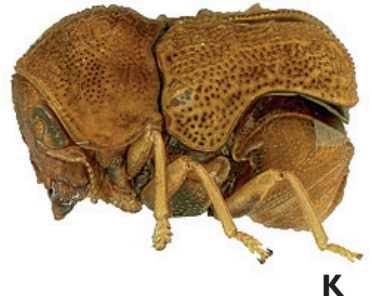

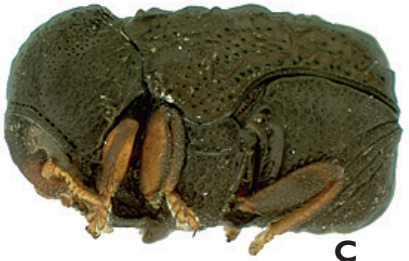

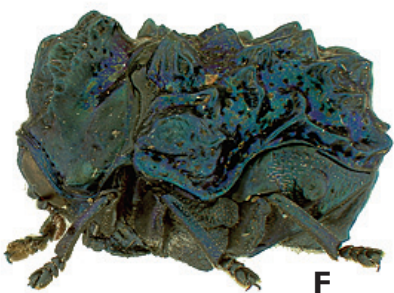

$\mathbf{F}$

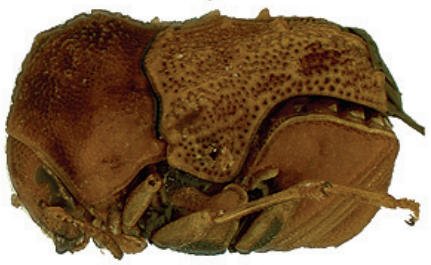

$\mathbf{L}$

Figure 2. Chlamisini, lateral view. A, Aulacochlamys distincta (Achard). B, A. costicollis (Lacordaire). C, Chlamisus foveolatus (Knoch). D, Diplacaspis prosternalis (Schaeffer). E, Exema elliptica Karren. F, Fulcidax coelestina (Lacordaire). G, Hymetes javana Lacordaire. H, Melittochlamys specula (Klug). I, Neochlamisus insularis (Schaeffer). J, N. velutinus Karren. K, Pseudochlamys megalostomoides Lacordaire $\delta$. L, P. megalostomoides ․ 
roico. II.952, coll. F. Monrós/ b. F. Monrós Collection, 1959. 3) a. Argent., Misiones, S. Sauier, P. Londero, Monrós, IX.947/ b. F. Monrós Collection, 1959. Aulacochlamys distincta (Achard):

1) a. Panason, Philippines/ b. F. Monrós Collection, 1959. 2) a. Manila, Philippines/ b. F. Monrós Collection, 1959/ c. Aulacochlamys distincta Achard. Aulacochlamys malayanus (Baly):

1) a. Sumatra, Benculen, IV. 1891, E. Modigliani/ b. F. Monrós Collection, 1959/ c. Exema malayana Baly/ d. Aulacochlamys malayanus Baly, F. Monrós det. 1955.

Aulacochlamys pygidialis Monrós:

1) a. Peru, Firgo, Moria, Kuschel, XII. 1946/ b. paratipos/ c. F. Monrós Collection, 1959/ d. Aulacochlamys pygidialis Monrós.

Aulacochlamys radiatus Monrós:

1) a. [Argentina] R.A. Misiones, A. Ogloblin leg./ b. paratipos/ c. F. Monrós Collection, 1959/ d. Aulacochlamys radiatus Monrós.

\section{Carcinobaena Lacordaire}

(Figs. $1 \mathrm{C} ; 3 \mathrm{~B} ; 4 \mathrm{~B} ; 5$ B)

Carcinobaena Lacordaire, 1848: 647. Type species: Chlamys pilula Klug 1824, by monotypy; Clavareau, 1913: 210 (catalog); Blackwelder, 1946: 647 (catalog); Monrós, 1952: 515 (detailed morphological study); Seeno and Wilcox, 1982: 43 (catalog).

Diagnosis. Length 4.54-5.36 mm, width 3.63-4.27 mm. General body shape subglobular. Antenna serrate beyond $6^{\text {th }}$ antennomere, $3^{\text {rd }}, 4^{\text {th }}$, and $5^{\text {th }}$ antennomeres slightly widened, but not dilated distally. Pronotum without median elevation, relatively smooth and continuous with rest of body, without well developed median longitudinal sulci. Pronotal base opposite mesoscutellum (posterior pronotal lobe) with notch. Prosternum broadly narrowing posteriorly, at apex not much narrower than anterior section of prosternum. Narrowest part of prosternal process at about $1 / 2$ length of entire prosternum. Mesoscutellum transverse, rectangular. Metascutellum concealed by elytra. Sutural serration of elytra poorly developed with individual serra wide, short, and rounded. Elytral tubercules not well developed. Femora and tibiae flattened; tarsi reduced, retractile, able to fit into groove along apex of tibia. Protibial apex with short medially facing spine. Midtibial apices without spine. Tarsal claw simple.

Distribution. The only species, Carcinobaena pilula (Klug), occurs in French Guiana and northern Brazil (Monrós, 1952).

Remarks. This genus resembles Melittochlamys in overall body shape; however, the modified legs of Carcinobaena immediately distinguish it not only from other chlamisines, but from most other genera in Chrysomelidae. 

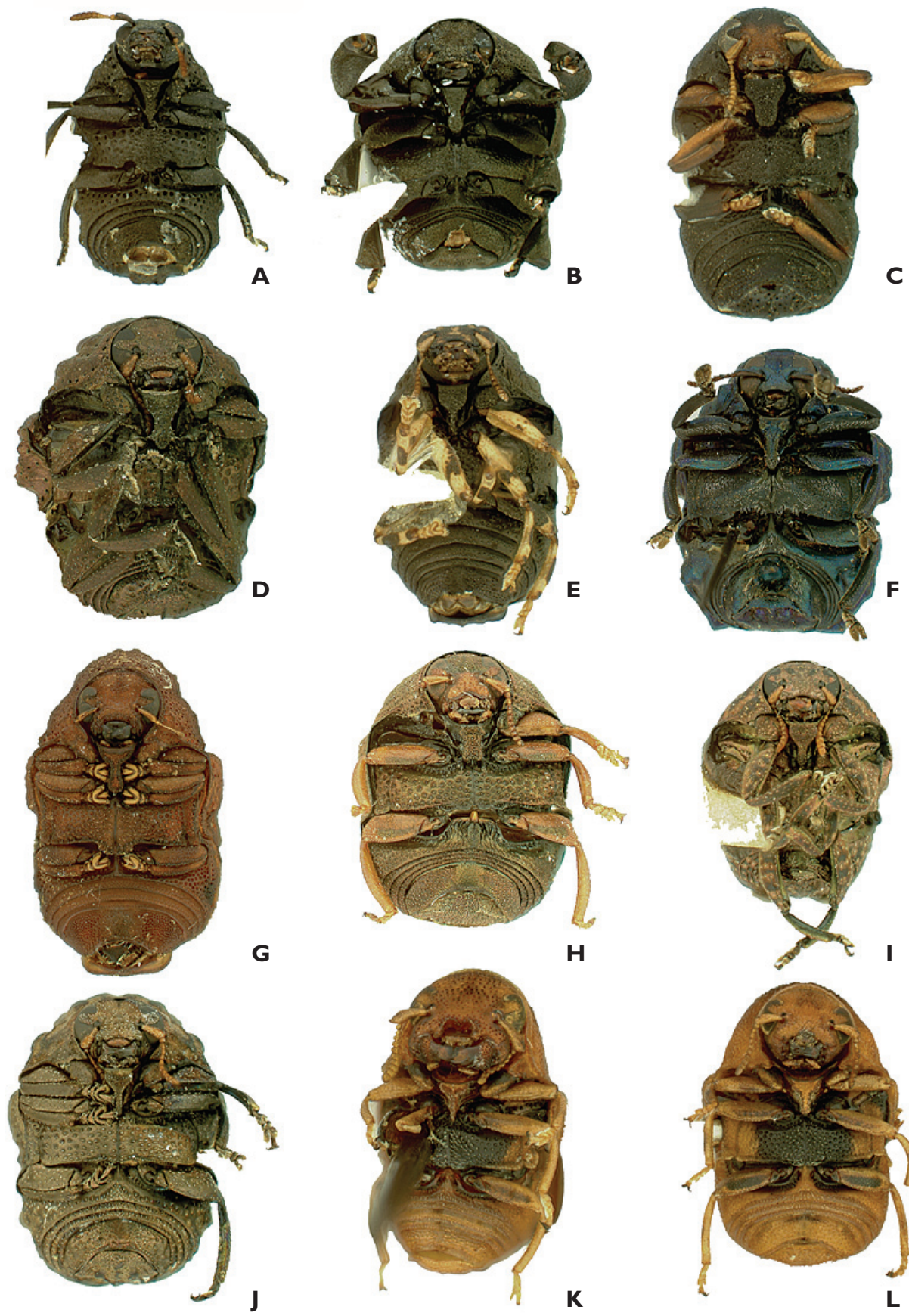

H

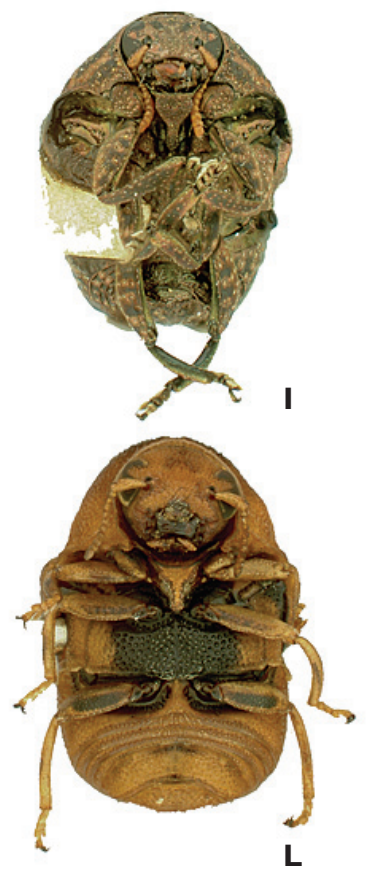

Figure 3. Chlamisini, ventral view. A, Aulacochlamys costicollis (Lacordaire). B, Carcinobaena pilula (Klug). C, Chlamisus foveolatus (Knoch). D, Diplacaspis prosternalis (Schaeffer). E, Exema elliptica Karren. F, Fulcidax coelestina (Lacordaire). G, Hymetes javana Lacordaire. H, Melittochlamys specula (Klug). I, Neochlamisus insularis (Schaeffer). J, N. velutinus Karren. K, Pseudochlamys megalostomoides Lacordaire $\delta$. L, P. megalostomoides q. 


\section{Material examined.}

Carcinobaena pilula (Klug):

1) a. Brazil, Para, Santarem: Tanerinha, VII.1921, I. Boy/ b. F. Monrós Collection, 1959/ c. Carcinobaena pilula (Klug), F. Monrós det. 1949. 2) a. Para, Brazil, de Mathan/ b. F. Monrós Collection, 1959. 3) a. Santarem, Brazil, F. Knab./ b. Carcinobaena pilula Klug/ c. Carcinobaena pilula (Klug), F. Monrós det. 1949.

\section{Chlamisus Rafinesque}

(Figs. 1 D; 2 C; 3 C; 4 C; 5 C; 7 B, D)

Chlamys Knoch, 1801: 122. Type species: Chlamys foveolata Knoch, 1801, by subsequent designation of Navajas, 1946: 244 [preoccupied by Chlamys Bolten, 1798, Mollusca]. Chlamisus Rafinesque, 1815: 116 (new name for Chlamys Knoch).

Diagnosis. Length 3.09-7.72 mm, width 1.90-5.36 mm. General body shape cylindrical. Body usually not metallic in color. Frons in canthus of eye usually without yellow spots (face may be entirely or partly yellow, with yellow area extending into canthus, but elytra do not have velvety spots). Frons glabrous or (rarely) covered with dense hairs. Pronotum and elytra usually glabrous or (rarely) covered with dense short hairs. Antenna serrate beyond $3^{\text {rd }}$ or $4^{\text {th }}$ antennomeres, $2^{\text {nd }}$ antennomere slightly widened, globose, $5^{\text {th }}$ antennomere nearly as large as $6^{\text {th }}$. Pronotum medially elevated, with various bumps and short carinae. Pronotal base opposite mesoscutellum (posterior pronotal lobe) with well differentiated notch. Prosternum posteriorly narrowed, posteriorly much narrower than anterior margin. Anterior margin of metasternum concave. Mesoscutellum short, transverse. Metascutellum not exposed. Sutural serration of elytra usually incomplete (suture entire immediately following mesoscutellum). Elytral tubercules well developed. Males without spines or spinulae on first ventrite. Tibiae slightly curved, slightly flattened, with sharp dorsal edge. Fore- and midtibial apices with spine. Tarsal claw appendiculate. Male ejaculatory guide (part of internal sac of aedeagus) symmetrical, without sheath. Apex of spermathecal duct as wide as rest of pump.

Distribution. Cosmopolitan (Monrós 1952; Reid 1991).

Remarks. Chlamisus is close to Exema and Neochlamisus. From Neochlamisus it can be separated by the following characters: body usually not metallic in color; frons in canthus of eye usually without yellow spots (face may be all or partly yellow, with yellow area extending into canthus, but elytra do not have velvety spots); and male ejaculatory guide symmetrical, without sheath. From Exema it can be separated by the following characters: males without spines or spinulae on first ventrite; $5^{\text {th }}$ antennomere nearly as large as $6^{\text {th }}$; sutural serration of elytra usually incomplete (suture entire immediately following mesoscutellum); and prosternum posteriorly pointed (narrowed), posteriorly much narrower than anterior margin.

No Australasian species were examined for this study, but according to Reid (1991) native Australasian species of Chlamisus do not fit into any current definition given 
for either American (Karren 1972; Monrós 1951) or Oriental (Gressitt and Kimoto, 1961) species. Reid (1991) also recognized several characteristics distinguishing Oriental (South-East Asian) and American species.

Comparison of the paratype of Chlamisus rousei Medvedev, 1993 described from Puerto Rico with specimens of Chlamisus straminea Suffrian, 1866 from Puerto Rico and Virgin Islands revealed that they are conspecific, therefore we here synonymize these names.

There are approximately 400 species of Chlamisus in the world (Monrós 1952; Reid 1991); approximately 100 Neotropical, 45 Palearctic, 18 Nearctic, 22 Oriental, 6 Afrotropical, and 2 Australasian (naturally occurring).

\section{Material examined.}

Chlamisus foveolatus (Knoch):

1) a. [USA] Beltsville, MD, VIII. 5/ b. Chlamisus foveolatus (Knoch) det. Karren 1971. 2) a. [USA] VI.10.11, Southern Pines, NC, AH Manee/ b. Chlamisus foveolatus (Knoch) det. Karren 1971.

Chlamisus maculipes (Chevrolat):

1) a. Mexico, Salle/ b. F. Monrós Collection, 1959/ c. Chlamisus maculipes (Chevr.) F. Monrós det. 1949.

Chlamisus obidensis Monrós:

1) a. [Brazil] Teffe (Ega), Amazonas, M. de Mathan, IV.78 et I.79/ b. F. Monrós Collection, 1959.

Chlamisus pilifrons (Lefevre):

1) a. [India] Rhamnaq (Hindustan)/ b. Ex. Musaeo Lefevre, 1894/ c. F. Monrós Collection, 1959/ d. Chlamisus pilifrons Lef. F. Monrós det. 1956.

Chlamisus pubiceps Chen:

1) a. Russia, Far East, Ussury region, Kondrat'evka, 28.VII.1992, leg. A. Konstantinov/ b. Chlamisus pubiceps Chen, det. A. Konstantinov.

Chlamisus semirufus Chen:

1) a. nr. Foochow, China, 1921-4, CR Kellog.

Chlamisus straminea Suffrian:

1) a. [Puerto Rico] on leaves of Diascorea, Ponce PR, 11.VIII.33, R.G. Oakley, SS 4489. 2) a. St. Thomas Vir. Ids., June 5, 1917 (122), Harold Morrison/ b. F. Monrós Collection, 1959/ c. Chlamisus straminea Suffr. F. Monrós det. 1954. 3) a. From Puerto Rico/ b. Paratypus, Chlamisus rousei L. Medv.

\section{Diplacaspis Jacobson}

(Figs. 1 E; 2 D; 3 D; 4 D; 5 D)

Diaspis Lacordaire, 1848: 645; Type species: Diaspis paradoxa Lacordaire, 1848, by monotypy; Jacoby, 1881: 74; Diaspsis Jacoby, 1889: 155 (misspelling; summary); [preoccupied by Diaspis Costa, 1835, Coccidae]

Diplacaspis Jacobson, 1924: 239 (new name for Diaspis Lacordaire); Blackwelder, 1946: 647 (catalog); Seeno and Wilcox, 1982: 43 (catalog); Riley et al., 2003: 183 (catalog). 


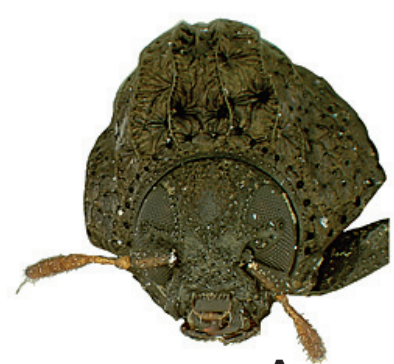

A

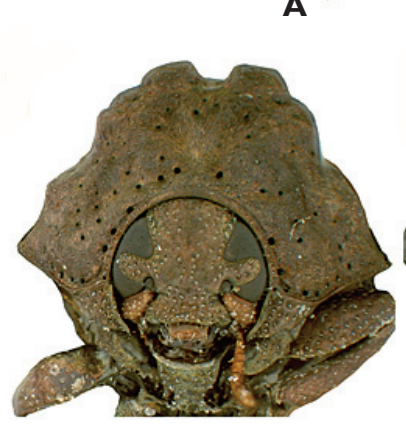

D

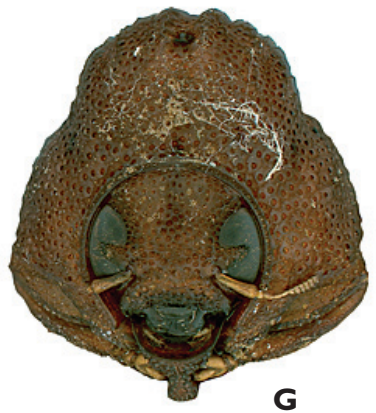

G

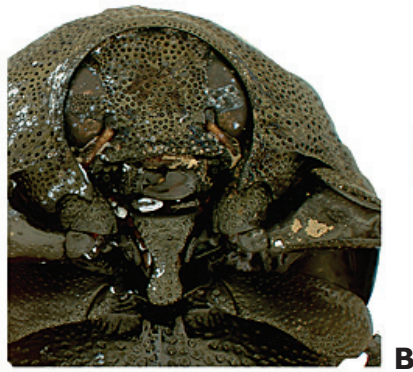

$\mathrm{B}$
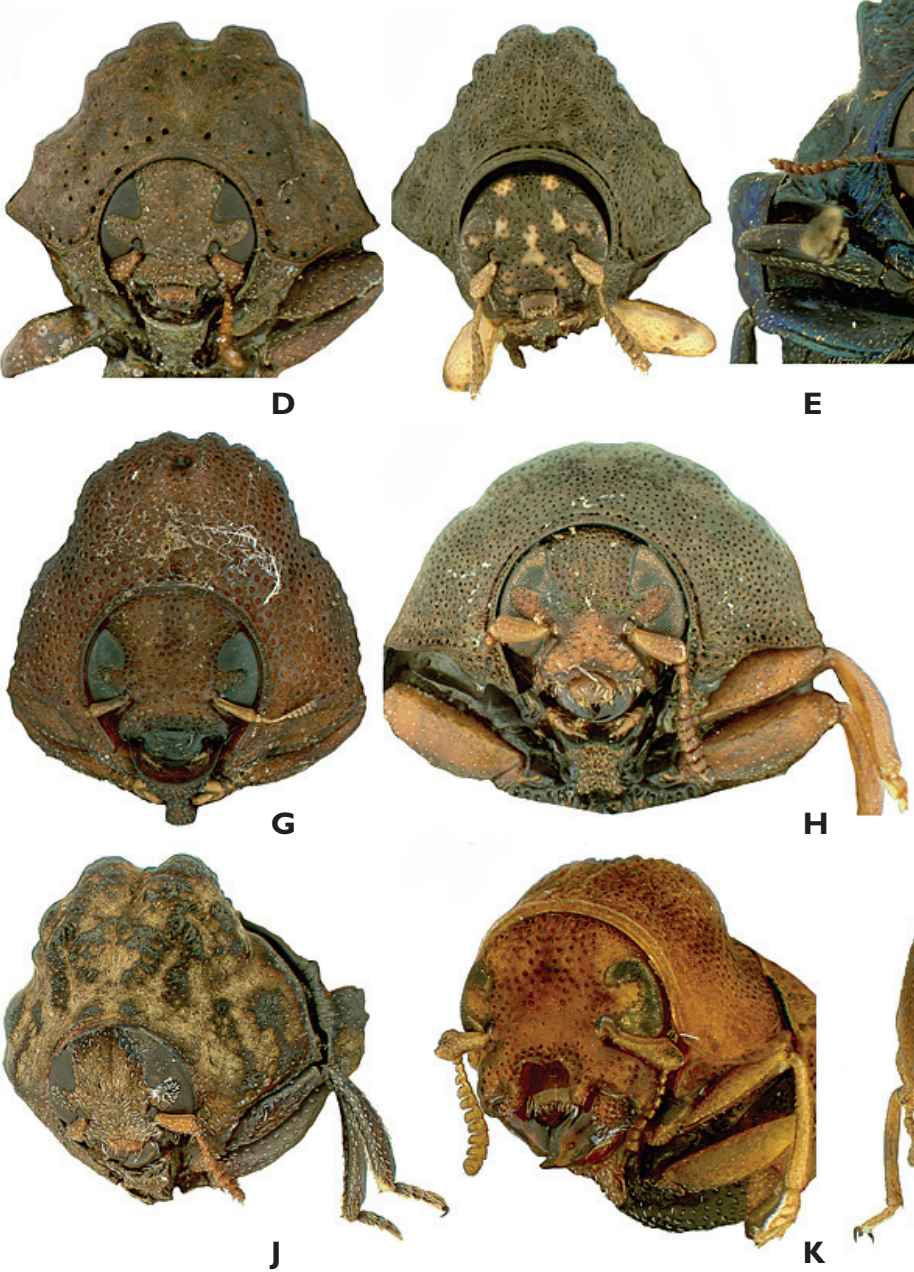

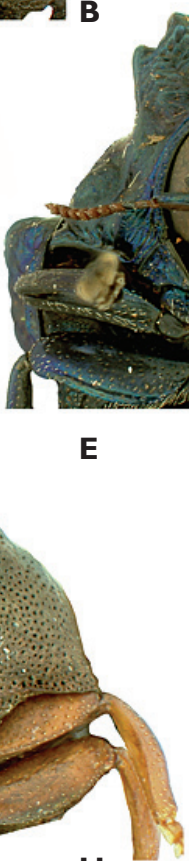

$\mathbf{H}$

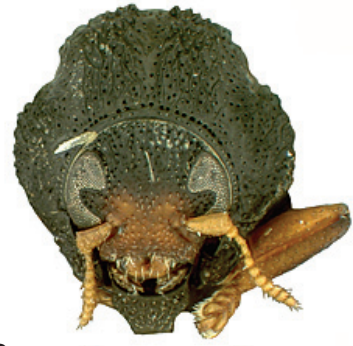

C
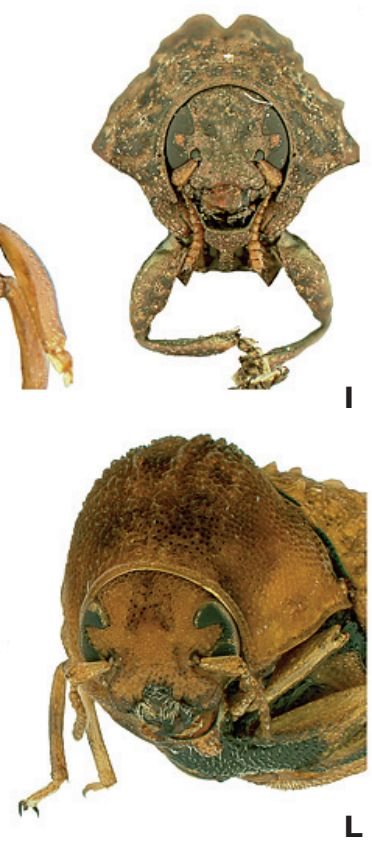

Figure 4. Chlamisini, frontal view. A, Aulacochlamys costicollis (Lacordaire). B, Carcinobaena pilula (Klug). C, Chlamisus foveolatus (Knoch). D, Diplacaspis prosternalis (Schaeffer). E, Exema elliptica Karren. F, Fulcidax coelestina (Lacordaire). G, Hymetes javana Lacordaire. H, Melittochlamys specula (Klug). I, Neochlamisus insularis (Schaeffer). J, N. velutinus Karren. K, Pseudochlamys megalostomoides Lacordaire ô. L, P. megalostomoides $q$. 
Skwarraia van Emden, 1932: 9 (new name for Diaspis Lacordaire).

Diagnosis. Length 3.00-3.81 mm, width 2.36-3.18 mm. General body shape subcylindrical, widest near shoulders. Antenna serrate beyond $4^{\text {th }}$ antennomere, $3^{\text {rd }}$ and $4^{\text {th }}$ antennomeres slightly widened, but not dilated distally. Pronotum medially elevated, with two tall projections separated by furrow. Pronotal base opposite mesoscutellum (posterior pronotal lobe) with wide notch. Prosternum gradually narrowing posteriorly, abruptly narrowing before middle, widening between mesocoxae. Anterior margin of metasternum concave. Mesoscutellum short, transverse. Metascutellum broadly exposed. Elytral suture serrate, entire (not serrate) in part that exposes metascutellum. Elytral tubercules well developed. Tibiae slightly curved, flattened, with sharp dorsal edge. Fore- and midtibial apices with spine. Tarsal claw appendiculate.

Distribution. New World (Monrós, 1952).

Remarks. This genus may be distinguished from other Chlamisini by the shape of the prosternum, and an exposed metascutellum in combination with the appendiculate tarsal claw. According to Karren (1972), South American species of Diplacaspis may not possess bifid claws or a posteriorly expanded prosternal process between the mesocoxae. However, the only two species available for our study [D. batesi (Baly) and D. prosternalis (Schaeffer)] share character states consistent with the diagnosis.

A total of 6 species currently are known in this genus (Monrós, 1952).

\section{Material examined.}

Diplacaspis batesi (Baly):

1) a. Brazil, Minas Gerais, col. M. Pic/ b. F. Monrós Collection, 1959/ c. Diplacaspis batesi (Baly) F. Monrós det. 1950.

Diplacaspis prosternalis (Schaeffer):

1) a. [Mexico] EA Schwarz Collector/ b. Monterrey, Mex. 25.II/ c. Diplacaspis prosternalis (Schaeffer) det. Karren 1968. 2) a. Granada, Nicaragua, Coll. Baker/ b. Diplacaspis prosternalis (Schaeffer) det. Karren 1968.

\section{Exema Lacordaire}

(Figs. 1 F; 2 E; 3 E; 4 E; 5 E)

Exema Lacordaire, 1848: 844; Type species: Chlamys intricata Kollar, 1824, by subsequent designation of Jacoby, 1908: 278; Karren, 1966: 1 (revision of North American species north of Mexico).

Diagnosis. Length 2.18-3.45 mm, width 1.36-2.72 mm. General body shape cylindrical, widest near shoulders. Antenna serrate beyond $5^{\text {th }}$ antennomere, $3^{\text {rd, }} 4^{\text {th }}$, and $5^{\text {th }}$ antennomeres slightly widened, but not dilated distally. Pronotum medially elevated, with various bumps and short ridges. Pronotal base opposite mesoscutellum (posterior pronotal lobe) concave, usually without well differentiated notch. Prosternum gradually narrowing posteriorly, at about middle abruptly narrowing to thin parallel sided 

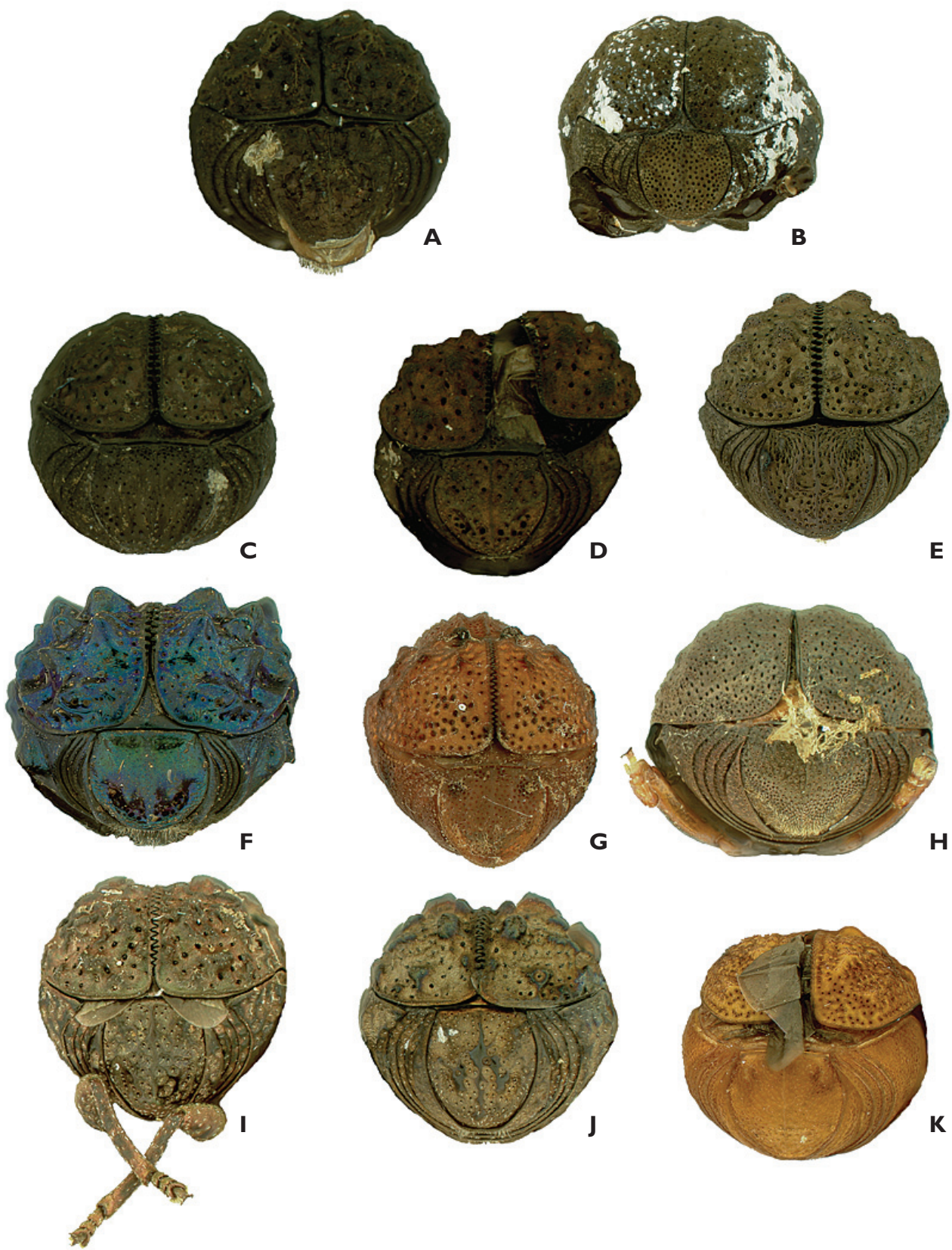

Figure 5. Chlamisini, caudal view. A, Aulacochlamys costicollis (Lacordaire). B, Carcinobaena pilula (Klug). C, Chlamisus foveolatus (Knoch). D, Diplacaspis prosternalis (Schaeffer). E, Exema elliptica Karren. F, Fulcidax coelestina (Lacordaire). G, Hymetes javana Lacordaire. H, Melittochlamys specula (Klug). I, Neochlamisus insularis (Schaeffer). J, N. velutinus Karren. K, Pseudochlamys megalostomoides Lacordaire . . 
ridge. Anterior margin of metasternum concave. Mesoscutellum short, transverse. Metascutellum not exposed. Elytral suture serration complete. Elytral tubercules well developed. Males with spines or spinulae on first ventral abdominal segment. Tibiae slightly curved, flattened, with sharp dorsal edge. Fore- and midtibial apices with spine in males, but lacking them in females. Tarsal claw from simple to appendiculate. Ejaculatory guide (part of internal sac of aedeagus) short, tubular, sclerotized.

Distribution. Nearctic, Neotropical, and Oriental Regions (Monrós, 1952; Karren, 1966).

Remarks. This genus may be distinguished from Chlamisus by the following characters: males with spines or spinulae on first ventral abdominal segment; $5^{\text {th }}$ antennomere much smaller than $6^{\text {th}}$; sutural serration of elytra complete (in Chlamisus males without spines or spinulae on first ventrite and $5^{\text {th }}$ antennomere nearly as large as $6^{\text {th }}$; sutural serration of elytra usually incomplete); ejaculatory guide short, single, tubular, and sclerotized (elongate and paired in Chlamisus). Lacordaire (1848) established Exema for species of Chlamisus that have a very abrupt antennal "club", the first serrated antennomere $\left(6^{\text {th }}\right)$ is much wider than $5^{\text {th }}$.

Exema was designated a junior synonym of Chlamisus by Gressitt and Kimoto (1961) based on their study of Oriental species; however, Exema continues to be treated as valid (Karren 1966, 1972; Riley et al. 2003; Seeno and Wilcox 1982).

A total of 26 species are included in Exema; 9 Nearctic, 7 Oriental, and 10 Neotropical (Monrós 1952; Karren 1966).

Material examined.

Exema canadensis Pierce:

1) a. [USA] Lakehead Md, 21.VII.06/ b. Ex. Coll. Knab/ c. Exema canadensis Pierce det. Karren 1966.

Exema elliptica Karren:

1) a. [USA] Houston, Tex. 25.V.49, T. L. Ward, Baccaharis halinifolial b. Paratype Exema elliptica Karren.

Exema variopicta Monrós:

1) a. [Argentina] R.A. Salta, San Bernardo, 30.I.950, Willink \& Monrós/ b. 1181/ c. F. Monrós Collection, 1959/ d. Exema variopicta Monrós F. Monrós det. 1954 .

\section{Fulcidax Voet}

(Figs. 1 G; 2 F; 3 F; 4 F; 5 F)

Fulcidax Voet, 1806: 33; Type species: Fulcidax azureus Voet, 1806 = Clytra monstrosa Fabricius, 1798, by monotypy; Jacoby, 1881: 90 (Central American species); Monrós, 1952: 641 (overview of genus); Blackwelder, 1946: 650 (catalog); Seeno and Wilcox, 1982: 43 (catalog).

Poropleura Lacordaire, 1848: 863; Type species: Clytra monstrosa Fabricius, 1798, by subsequent designation of Navajas, 1946: 245. 
Diagnosis. Length $6.50-7.20 \mathrm{~mm}$, width $4.60-5.00 \mathrm{~mm}$. General body shape subquadrate, metallic coloration. Antenna serrate beyond $3^{\text {rd }}$ antennomere, $2^{\text {nd }}$ and $3^{\text {rd }}$ antennomeres slightly widened, but not dilated distally. Head with the vertex longitudinally impressed. Pronotum with median elevation, surface tuberculate. Pronotal base opposite mesoscutellum (posterior pronotal lobe) with acute notch. Prosternum acutely narrowing posteriorly, prosternal process $2 / 3$ size of entire prosternum. Anterior margin of metasternum concave. Mesoscutellum trapezoidal. Metascutellum not exposed. Sutural serration of elytra well developed beyond middle of suture towards the apex. Elytral tubercules pronounced. First ventrite with lateral tubercles. Tibiae slightly curved, flattened, with sharp dorsal edge. Fore- and midtibial apices with spine. Tarsal claw simple.

Distribution. Central and South America (Monrós 1952).

Remarks. Fulcidax can be distinguished from all other chlamisine genera by the longitudinally impressed vertex of the head, simple tarsal claws, large body size, and usually bright metallic coloration.

Currently, 7 species are included in the genus (Monrós 1952).

Material examined.

Fulcidax bacca (Kirby):

1) a. [Brazil] Canlareira, S. Paulo, 8.VII.1929, J. Halik, 1275/ b. Fulcidax bacca

Kirby/ c. J. Guerrin det. 1942/ d. Brazil, Halik 1966 Collection. 2) a. Brazil/ b.

CF Baker collection, 1927/ c. Fulcidax bacca (Kirby) F. Monrós det. 1949.

Fulcidax chimaera (Lacordaire):

1) a. [Argentina] Munici[pi]o Rio Verde, Estado Goyaz, Dr. Nick. XI.945/ b.

F. Monrós Collection, 1959/ c. Fulcidax chimaera (Lac.) F. Monrós det. 1952. Fulcidax coelestina (Lacordaire):

1) a. Bolivia, Trinidad, X.1917 Lizer. Deletang/ b. F. Monrós Collection, 1959/ c. Fulcidax coelestina (Lac.) F. Monrós det. 1948.

\section{Hymetes Lacordaire}

(Figs. 1 H; 2 G; 3 G; 4 G; 5 G)

Hymetes Lacordaire, 1848: 861; Type species: Hymetes javana Lacordaire, 1848, by monotypy; Baly, 1865: 61 (description)

Diagnosis. Length 6.34-7.10 mm, width 4.27-4.46 mm. General body shape subquadrate. Antenna serrate beyond $5^{\text {th }}$ antennomere, $2^{\text {nd }}$ antennomere globular, $3^{\text {rd }}, 4^{\text {th }}$ and $5^{\text {th }}$ antennomere of equal size, subcylindrical, $6^{\text {th }}$ antennomere very short. Pronotum medially elevated, without pronounced tubercules, but with relatively short, acute ridges. Pronotal base opposite mesoscutellum (posterior pronotal lobe) with acute notch. Prosternal process approximately $1 / 2$ length of entire prosternum, narrow, slightly broadening posteriorly. Anterior margin of metasternum forming an elongate projection. Mesoscutellum trapezoidal. Metascutellum not exposed. Sutural serra- 
tion of elytra complete. Elytral tubercules weakly developed. Tibiae slightly curved, flattened dorsally, with sharp edge dorsomedially. Fore- and midtibial apices without spine. Tarsal claw appendiculate.

Distribution. Oriental Region (Monrós 1952).

Remarks. This genus can be separated from all other chlamisine genera by the anteriorly elongate metasternum projection (concave in other chlamisines), by the subcylindrical $3^{\text {rd }}, 4^{\text {th }}$ and $5^{\text {th }}$ antennomeres, and by the absence of spines on the fore- and midtibiae.

Three species are known from India and Java (Monrós 1952).

\section{Material examined.}

Hymetes javana Lacordaire:

1) a. [Indonesia] Java/ b. F. Monrós Collection, 1959. 2) a. [Indonesia] East Borneo, Batan bessi, M. E. Walsh, 1937/ b. F. Monrós Collection, 1959/ c. Hymetes javana Lac. F. Monrós det. 1957.

Hymetes indica Chapuis:

1) a. India/ b. F. Monrós Collection, 1959/ c. Hymetes indica Chap F. Monrós det. 1957.

\section{Kakita Chamorro-Lacayo \& Konstantinov, new name}

(Fig. 6 A-E)

Ceratochlamys Bokermann, 1961:465, nec Habe, 1946: 214 (Mollusca); Type species: Ceratochlamys monrosi Bokermann, 1961, by monotypy; Seeno and Wilcox, 1982: 43 (catalog).

Diagnosis. Length $5.20 \mathrm{~mm}$, width $2.70 \mathrm{~mm}$. General body shape oblong. Head with large laminar projection on occipital region, projection longer than entire head. Antenna serrate beyond $5^{\text {th }}$ antennomere, $3^{\text {rd }}$ antennomere elongate, cylindrical, $4^{\text {th }}$ antennomere slightly dilated distally. Pronotum large with paired pyramidal projections directed towards cephalic projection. Prosternal process $2 / 3$ length of entire prosternum, narrow. Mesoscutellum trapezoidal. Sutural serration of elytra incomplete. Elytral tubercules well developed. Tarsal claws appendiculate.

Distribution. The only species, Kakita monrosi (Bokermann), is known from Rio de Janeiro, Brazil (Bokerman 1961).

Remarks. Only a single female specimen represents this genus. Whether the laminar projection on the head, which distinguishes this genus from all other chlamisines, is sexually dimorphic is unknown. In leaf beetles, sexually dimorphic characters on the head, such as projections and enlarged mandibles, are usually present only on the male (e.g., Labidostomis Germar; Pseudochlamys Lacordaire; Normaltica Konstantinov) (Konstantinov 2004; Konstantinov and Korotyaev 2004). On the other hand, all female cryptocephalines have a modified $5^{\text {th }}$ ventrite for the purpose of scatoshell coating. It is possible, that this laminar projection on the head is only present on the female 

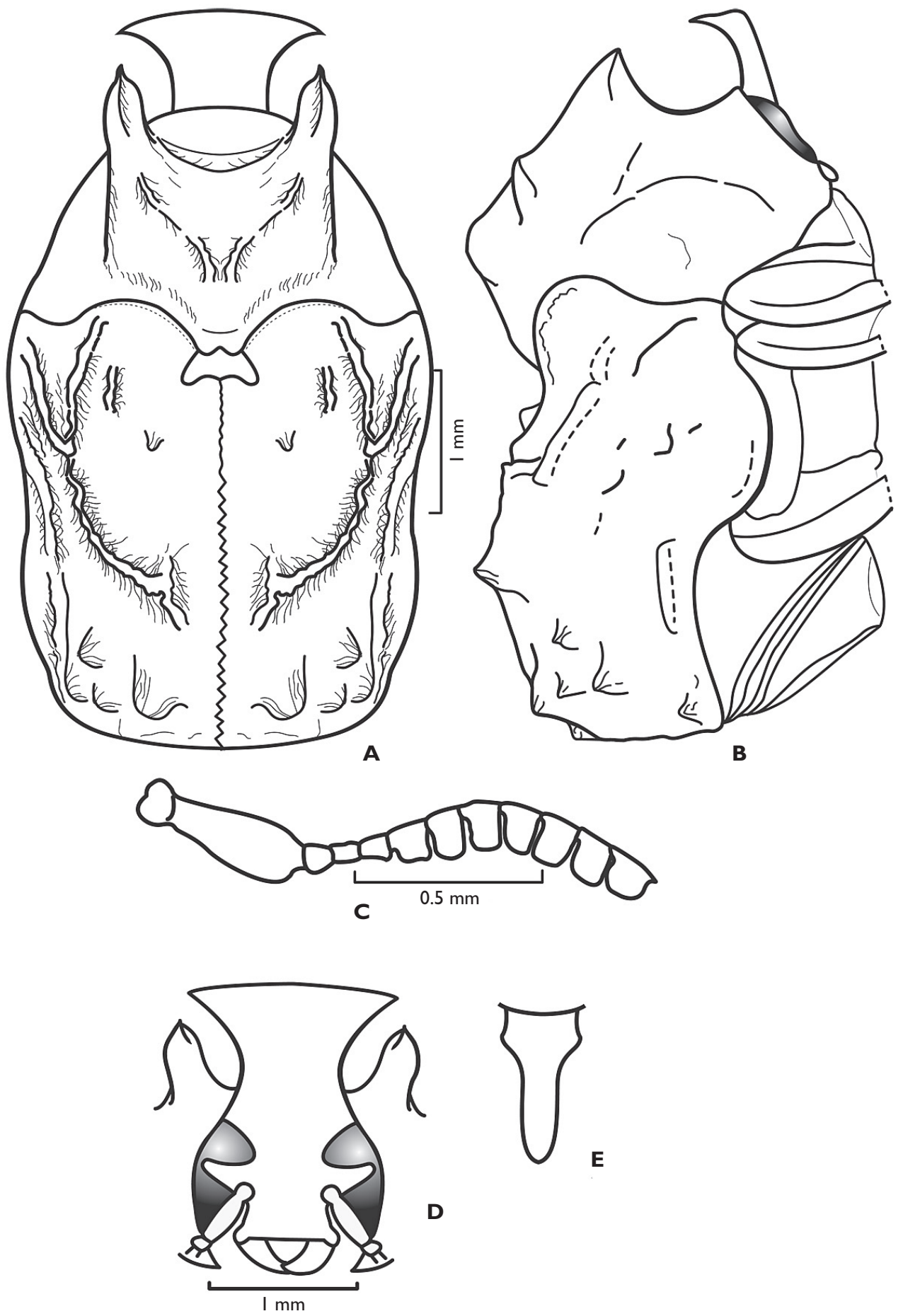

Figure 6. Kakita monrosi (Bokermann). A, Ventral view. B, Lateral view. C, Antenna. D, Frontal view. E, Prosternum. 
also for purposes dealing with coating of individual eggs. Females, during the coating process, strike a pose in which the entire weight of their bodies rests on their forelegs and possibly their head. This laminar projection may be helpful in balancing their body. One thing is certain, observation in the field and continued collecting efforts to discover the male are necessary to understand the function of this modification.

The name Ceratochlamys Bokermann, 1961 is preoccupied by an available name in Mollusca, Ceratochlamys Habe, 1946. A replacement name, Kakita Chamorro-Lacayo \& Konstantinov is provided and Ceratochlamys Bokermann is invalid as a junior homonym.

Etymology. Kakita, modified from its correct spelling "caquita", is a Spanish word for "small feces or excrement". The name alludes to the resemblance these beetles have to small pieces of excrement. The name is feminine.

\section{Melittochlamys Monrós}

(Figs. $1 \mathrm{I} ; 2 \mathrm{H} ; 3 \mathrm{H} ; 4 \mathrm{H} ; 5 \mathrm{H}$ )

Melittochlamys Monrós, 1948: 192; Type species: Chlamys speculum Klug, 1824, by original designation; Fiebrig, 1910: 253 (larval description); Monrós, 1949: 617 (description of new species and generic concept broadened); Monrós, 1951: 451 (key and description of new species); Monrós, 1952: 666 (generic overview); Seeno and Wilcox, 1982: 43 (catalog).

Diagnosis. Length 3.60-5.20 mm, width 2.60-4.00 mm. General body shape subglobular. Antenna serrate beyond $3^{\text {rd }}$ antennomere, $3^{\text {rd }}$ antennomere only slightly dilated distally. Pronotum without median elevation, relatively smooth and continuous with rest of body, without well developed median longitudinal sulci. Pronotal base opposite mesoscutellum (posterior pronotal lobe) with or without notch. Prosternum not acutely narrowing posteriorly, prosternal process broad and parallel-sided. Anterior margin of metasternum broadly concave. Mesoscutellum quadrate. Metascutellum not exposed. Sutural serration of elytra completely absent or weakly developed. If sutural elytral serration present, well developed beyond middle of suture towards the apex. Elytral tubercules not well developed, frequently with velvety, discrete spots and microsculpture different from rest of body surface. Tibiae slightly curved, convex dorsally, with sharp edge dorsomedially. Fore- and midtibial apices without spine. Tarsal claw appendiculate.

Distribution. Central and South America (Monrós 1952).

Remarks. Some species of Chlamisus (e.g., Chlamisus achalay Monrós, 1952 and Chlamisus perforatus Monrós, 1952) also have velvety spots on the elytra while others have a broad prosternal process. Monrós (1949) broadened his own definition of the genus to include species that lack velvety spots on the elytra, have a broad, parallelsided prosternal process, and a globous, oval body shape with the pronotum dorsally smooth and continuous with rest of body. Melittochlamys can be separated from all other chlamisine genera by the nearly rectangular prosternal process; the process is more or less triangular in other chlamisines. 
The genus consists of 13 species from the Neotropical Region. These include the first seven species Lacordaire (1848) listed in his division I under Chlamisus Rafinesque, one species described by Jacoby (1889), and four species described by Monrós (1948, 1949, 1951), most recently Bokermann (1964) described a species from Pará, Brazil.

\section{Material examined.}

Melittochlamys lamprosomoides (Lacordaire):

1) a. Santarem. Brazil. F. Knab/ b. collection F Knab/ c. Chlamys lamprosomoides Lac./ d. Melittochlamys lamprosomoides (Lac.) F. Monrós det. 1949.

Melittochlamys nicki Monrós:

1) a. [Brazil] Jabaquara, San Paolo-Capital, Dr. Nick 14.12.43/ b. Paratipo/

c. Dibujado/ d. F. Monrós Collection, 1959/ e. Melittochlamys nicki mihi F. Monrós det. 1949.

Melittochlamys specula (Klug):

1) a. Loreto Misiones, Rep. Argentina, Dr. A. Ogloblin/ b. Dibujado/ c. F. Monrós Collection, 1959/ d. Melittochlamys specula (Klug) F. Monrós det. 1948.

\section{Neochlamisus Karren}

(Figs. 1 J, K; 2 I, J; 3 I, J; 4 I, J; 5 I, J; 7 A, C)

Neochlamisus Karren, 1972: 932; Type species: Neochlamisus velutinus Karren, 1972, by original designation.

Diagnosis. Length 2.90-4.70 mm, width 2.10-3.68 mm. General body shape cylindrical. Body usually metallic in color. Frons in canthus of eye usually with yellow spots, or if without yellow spots, then elytron with two velvety spots. Frons glabrous. Pronotum and elytra glabrous. Antenna serrate beyond $3^{\text {rd }}$ or $4^{\text {th }}$ antennomeres, $2^{\text {nd }}$ antennomere slightly widened, globose, $5^{\text {th }}$ antennomere smaller than $6^{\text {th }}$. Pronotum medially elevated, with various bumps. Pronotal base opposite mesoscutellum (posterior pronotal lobe) with well differentiated notch. Prosternum posteriorly narrow or explanate with lateral serration. Anterior margin of metasternum concave. Mesoscutellum short, transverse. Metascutellum exposed or concealed. Sutural serration of elytra usually incomplete (suture entire immediately beyond mesoscutellum, followed by well developed serration). Elytral tubercules well developed. Tibiae slightly curved, slightly flattened, with sharp dorsal edge. Fore- and midtibial apices with spine. Tarsal claw bifid or appendiculate. Male ejaculatory guide (part of internal sac of aedeagus) asymmetrical, with sheath. Apex of spermathecal duct globosely swollen, wider than rest of pump.

Distribution. North, Central, and South America (Karren 1972).

Remarks. Neochlamisus was proposed to include a few species, formerly placed in Chlamisus and Diplacaspis, based on their similarity in the male and female genitalia (Karren 1972). Degree of exposure of the metascutellum varies greatly, from broadly exposed to completely concealed by elytra. Neochlamisus can be separated from other 


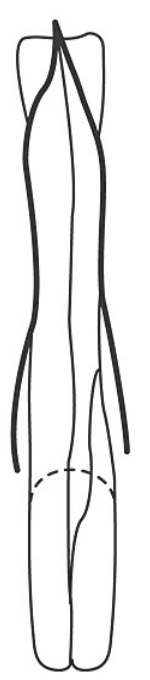

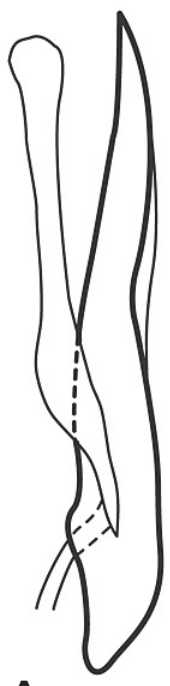

A

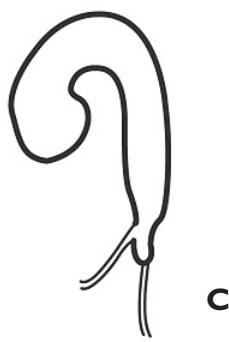

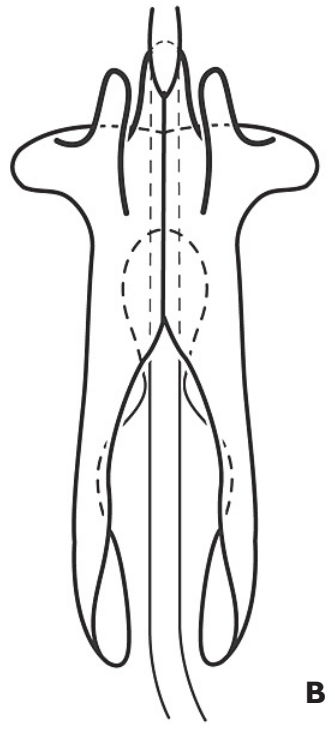
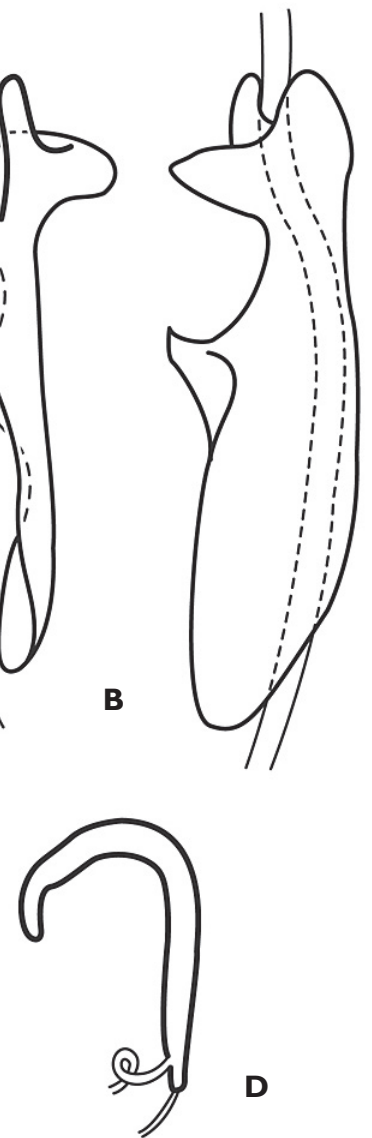

Figure 7. Genitalia of Chlamisini. A, Male ejaculatory guide of Neochlamisus velutinus Karren, dorsal and lateral views. B, Male ejaculatory guide of Chlamisus maculipes (Chevrolat), dorsal and lateral views. C, Spermatheca of Neochlamisus velutinus. D, Spermatheca of Chlamisus maculipes.

chlamisine genera by characters of the male genitalia. The male ejaculatory guide is asymmetrical, with a sheath. Among external characters, color of the frons in combination with the presence of velvety spots usually allows for recognition of Neochlamisus (frons on canthus of eye usually with yellow spots, or if without yellow spots, then elytron with two velvety spots).

\section{Material examined.}

Neochamisus cribripennis (LeConte):

1) a. [USA] Midvale N.J. A. Nicolay, 20.VIII.42/ b. Ernest Shoemaker Collection. 2) a. [Canada] Constance Bay, Ont., 22.V.42, W.J. Brown/ b. on Vaccinium/ c. Neochamisus cribripennis (LeConte) det. Karren 1968.

Neochlamisus insularis (Schaeffer):

1) a. [USA] Paradise Key, Fla. 25.II.19, EA Schwarz/ b. Neochlamisus insularis (Schaeffer) det. Karren. 
Neochlamisus tuberculatus (Klug):

1) a. [USA] Capron Fla. 8.4/ b. ex coll. Knabe/ c. 409/ d. Neochlamisus tuberculatus (Klug) det Karren 1971.

Neochlamisus velutinus Karren:

1) a. [USA] 8 mi San Vicente, Ariz. (Pima) 3000’ Aug.8.1954, F. G. Werner/

b. F. Monrós Collection, 1959/ c. Paratype Neochlamisus velutinus m. Jay B. Karren.

\section{Pseudochlamys Lacordaire}

(Figs. 1 L; 2 K, L; 3 K, L; 4 K, L; 5 K)

Pseudochlamys Lacordaire, 1848:644; Type species: Pseudochlamys megalostomoides Lacordaire, 1848, by monotypy; Clavareau, 1913: 209 (catalog); Blackwelder, 1946: 647 (catalog); Monrós, 1952: 542 (summary of characters and distribution); Karren, 1972: 902 (overview of genus and description of new species); Seeno and Wilcox, 1982: 43 (catalog).

Diagnosis. Length 3.45-4.72 mm, width 2.18-3.22 mm. General body shape cylindrical. Body usually yellowish. Frons glabrous, canthus of eye as yellow as rest of frons. Pronotum and elytra glabrous. Head not completely retracted into prothorax; mandibles enlarged in males, normal in females (Figs. $4 \mathrm{~K}, \mathrm{~L}$ ). Antenna serrate beyond $3^{\text {rd }}$ antennomere, $2^{\text {nd }}$ antennomere slightly widened, globose, $5^{\text {th }}$ antennomere as large as $6^{\text {th }}$. Pronotum medially elevated, with small protuberances. Pronotal base opposite mesoscutellum (posterior pronotal lobe) with well differentiated notch. Prosternum strongly and abruptly constricted beyond anterior margin; prosternal process more than $3 / 4$ as long as prosternum. Anterior margin of metasternum concave. Mesoscutellum short, transverse. Metascutellum concealed by elytra. Sutural serration of elytra complete. Elytral tubercules poorly developed, their microsculpture not different from rest of body surface. Tibiae slightly curved, more or less cylindrical, with sharp dorsal edge and one more less developed ventral ridge. Fore- and midtibial apices without spine. Tarsal claw bifid or appendiculate.

Distribution. North, Central, and South America (Karren 1972).

Remarks. Pseudochlamys can be distinguished from other chlamisines by the following characters: head not completely retracted into prothorax; mandibles enlarged in males, normal in females; fore- and midtibial apices without spine; prosternum strongly and abruptly constricted beyond anterior margin; and prosternal process more than $3 / 4$ as long as prosternum.

Five species are included in this genus.

Material examined.

Pseudochlamys bellicosus Monrós:

1) a. Brazil, Bahia, G. Bondar/ b. 2 paratypos/ c. F. Monrós Collection, 1959/

d. Pseudochlamys bellicosus mihi F. Monrós det. 1952. 
Pseudochlamys megalostomoides Lacordaire:

1) a. Brazil, Ceara, Ex. US.N.M./ b. F. Monrós Collection, 1959/ c. Pseudochlamys megalostomoides Lac. F. Monrós det. 1949. 2) a. Aguadulce, Panama, IX.1946/ b.

N.L.H. Krauss/ c. Pseudochlamys megalostomoides Lacordaire Det. Karren, 1971. Pseudochlamys seminigra (Jacoby):

1) a. Paraguay, Villarrica, Schade leg./ b. Dibujado/ c. F. Monrós Collection, 1959/ d. Pseudochlamys seminigra (Jac.) F. Monrós det. 1950.

Pseudochlamys semirufescens Karren:

1) a. USA, Ariz., Santa Catalina Mts. Pepper Sauce cn. 16.VIII.1924, E.P. Van

Duzee/ b. F. Monrós Collection, 1959/ c. Paratype Pseudochlamys semirufescens 1971 \& Jay B. Karren.

\section{Key to genera}

1 Head with large laminar projection in occipital region. (Figs. 6 A, B, D) ...... Kakita Chamorro-Lacayo \& Konstantinov, new name

- Head without large laminar projection in occipital region ...........................2

2(1) Legs atypical; femora and tibiae flattened; tarsi reduced, retractile, able to fit into groove along apex of tibia (Fig. 3 B) ... Carcinobaena Lacordaire, 1848

- $\quad$ Legs normal and not as above (Fig. $3 \mathrm{E}, \mathrm{H}$ ) .............................................. 3

3(1) Prosternum nearly rectangular, with posterior margin slightly narrower than anterior margin (Fig. $3 \mathrm{H}$ ). Pronotal and elytral tubercules reduced.

Melittochlamys Monrós, 1948

- $\quad$ Prosternum varying in shape, triangular, or angulate between mesocoxae, but never rectangular, with posterior margin much narrower than anterior margin (Figs. 3 A, I). Pronotal and elytral tubercules well developed ................ 4

4(3) Head with vertex longitudinally impressed. Tarsal claws simple. Body about $10 \mathrm{~mm}$ long and longer, bright metallic color. Pronotal and elytral tubercules pronounced Fulcidax Voet, 1806

- $\quad$ Head with vertex entire or convex. Tarsal claws usually appendiculate, if simple, see Exema. Body less than $10 \mathrm{~mm}$ long

5(4) Prosternal process distally widened between midcoxae (Fig. 3 D). Metascutellum broadly exposed (Fig. 1 E) ........................ Diplacaspis Jacobson, 1924

- $\quad$ Sides of prosternal process convergent or serrate, if slightly widened between mesocoxae, then metascutellum not exposed (Fig. 1 A)

6(5) Pronotum with 6 distinct, small, sharp, longitudinal carinae that converge medially near posterior margin (fan). Small size, elongate, uniform hue, generally black (Figs. $1 \mathrm{~A}, \mathrm{~B}$ ).

Aulacochlamys Monrós, 1952

- $\quad$ Pronotum with or without bumps, but never with 6 longitudinal carinae that converge medially near posterior margin.....

7(6) Head not completely retracted into the prothorax; mandibles in males larger than in females (Fig. $4 \mathrm{~K}, \mathrm{~L}$ ). Prosternum strongly and abruptly constricted 
beyond anterior margin; prosternal process more than $3 / 4$ as long as prosternum. (Figs. 3 K, L) ................................ Pseudochlamys Lacordaire, 1848

- Head completely retracted into prothorax; mandibles in males as large as in females (Fig. 4 A). Prosternum gradually constricted at about $2 / 3$ of its length

8(7) Metasternum anteriorly elongated into deltoid projection (Fig. $3 \mathrm{G}$ ). Antennae serrate beyond antennomere 5 Hymetes Lacordaire, 1848

- Metasternum anteriorly concave with two denticles on sides of concavity (Fig. 3 B).

9(8) Body usually metallic in color. Frons on canthus of eye usually with yellow spots, or if without yellow spots, then elytron with two velvety spots. Male ejaculatory guide asymmetrical, with sheath (Fig. 7 A)

Neochlamisus Karren, 1972

- $\quad$ Body usually not metallic in color. Frons on canthus of eye usually without yellow spots (if face entirely or partly yellow, with yellow area extending onto canthus, elytra without velvety spots). Male ejaculatory guide symmetrical, without sheath (Fig. 7 B)

10(9) Males without spines or spinulae on first ventrite. Fifth antennomere nearly as large as $6^{\text {th }}$. Sutural serration of elytra usually incomplete (suture entire immediately following mesoscutellum) (Fig. 1 D). Prosternum posteriorly pointed (narrowed), posteriorly much narrower than anterior margin

Chlamisus Rafinesque, 1815

- Males with spines or spinulae on first ventrite. Fifth antennomere much smaller than $6^{\text {th }}$. Sutural serration of elytra complete (Fig. 1 F). Pronotum without longitudinal carinae

Exema Lacordaire, 1848

\section{Acknowledgements}

We thank J. Brown and A. L. Norrbom (Systematic Entomology Laboratory, ARS, USDA, Washington, DC), and A. Tishechkin (Department of Entomology, Louisiana State University, Raton Rouge, LA) for reviewing earlier versions of this manuscript and providing valuable suggestions.

\section{References}

Baly JS (1865) Phytophaga Malayana; a revision of the phytophagous beetles of the Malay Archipelago, with descriptions of the new species collected by Alfred R. Wallace. Transactions of the Entomological Society London 3(4): 1-76.

Blackwelder RE (1946) Checklist of the Coleopterous Insects of Mexico, Central America, the West Indies, and South America. Smithsonian Institution, United States National Museum Bulletin 185: iii + 551-763. 
Bokermann WCA (1961) Um novo Gênero e uma nova Espécie de Chlamisinae (Col., Chrysomelidae). Studia Entomologica 4: 465-470.

Bokermann WCA (1964) Novos Chlamisinae Neotropicais (Coleoptera, Chrysomelidae) (20a Contribução). Revista Brasileira de Entomologia 11: 63-83.

Braga Sobrinho R, Mesquita ALM, Bandeira CT (1999) Occurrence of Fulcidax coelestina (Lac.) (Coleoptera: Chrysomelidae: Fulcidacinae) in Barbados cherry Malpighia glabra L. Anais da Sociedade Entomologica do Brasil 28: 541-542.

Brown CG, Funk DJ (2005) Aspects of the Natural History of Neochlamisus (Coleoptera: Chrysomelidae): Fecal Case-Associated Life History and Behavior, with a method for Studying Insect Constructions. Annals of the Entomological Society of America 98: 711-725.

Chaboo CS, Brown CG, Funk DJ (2008) Fecal case architecture in the gibbosus species group of Neochlamisus Karren, 1972 (Coleoptera: Chrysomelidae: Cryptocephalinae: Chlamisini). Zoological Journal of the Linnean Society 152: 315-351.

Chamorro-Lacayo ML, Konstantinov AS (2004) Comparative morphology of the prothorax and procoxa in the New World Cryptocephalini (Coleoptera: Chrysomelidae: Cryptocephalinae). Zootaxa 676: 1-46.

Chamorro-Lacayo ML, Konstantinov AS, Moseyko AG (2006) Comparative morphology of the female genitalia and abdominal structures of Neotropical Cryptocephalini (Coleoptera: Chrysomelidae: Cryptocephalinae). Coleopterists Bulletin 60: 113-134.

Chûjô M (1940) Fulcidacinae of Formosa (Col. Chrysomelidae). Transactions Natural History Society Formosa 30: 265-293.

Chûjô M (1942) Fulcidacinae of Japan proper and Loo-Choo (Coleoptera-Chrysomelidae). Transactions Natural History Society Formosa 32: 77-100.

Clavareau H (1913) Coleopterorum Catalogus, Chrysomelidae: Chlamydinae, pars. 53 (9): 209-222. W. Junk, Berlin.

Emden F van (1932) Die Lrven von Discoloma cassideum Reitt. (Col. Colyd.) und Skwarraia paradoxa Lac. (Col. Chrysom.). Zoologischer Anzeiger 101: 1-17.

Erber D (1988) Biology of Camptosomata Clytrinae - Cryptocephalinae - Chlamisinae Lamprosomatinae. In: Jolivet P, Petitpierre E, Hsiao TH (Eds) Biology of Chrysomelidae. Kluwer Academic Publishers, Dordcrecht/Boston/London, 513-552.

Fiebrig K (1910) Cassiden und Cryptocephaliden Paraguays. Ihre Entwicklungsstadien und Schutzvorrichtungen. Zoologische Jahrbücher Supplement 12: 161-264.

Gressitt JL, Kimoto S (1961) The Chrysomelidae (Coleopt.) of China and Korea. Part 1. Pacific Insects Monographs 1A: 1-299.

Habe T (1946) Reviews of Japanese Helicarionidae pt. 3. Venus 14. [in Japanese]

Jacobson G (1924) Annotationes synonymicae et systematicae de Coleopteris. Revue Russe d'Entomologie (Russkoe entomologicheskoe obozrenie) 18: 237-244.

Jacoby M (1881) Biologia Centrali-Americana, Insecta. Coleoptera. Phytophaga (part). Coleotpera, Cryptocephalidae, Chlamydidae, Lamprosomidae, Eumolpidae, vol. 6. R.H. Porter, London, 38-187.

Jacoby M (1889) Biologia Centrali-Americana, Insecta. Coleoptera. Supplement Phytophaga (part) 6(1): 81-168. 
Jacoby M (1908) The fauna of British India, including Ceylon and Burma. Coleoptera. Chrysomelidae. Vol. 1. Taylor and Francis, London, 534 pp.

Karren JB (1966) A revision of the genus Exema of America, north of Mexico (Chrysomelidae, Coleoptera). The University of Kansas Science Bulletin 46: 647-695.

Karren JB (1972) A revision of the subfamily Chlamisinae of America north of Mexico (Coleoptera: Chrysomelidae). The University of Kansas Science Bulletin 49: 875-988.

Knoch AW (1801) Neue Beyträge zur Insectenkunde, vol. 1. Schwinkert, Leipzig, 208 pp.

Konstantinov AS (2004) Male combat and mating behavior of Donacia crassipes Fabricius and other chrysomelids (Coleoptera, Chrysomelidae, Donaciinae). In: Jolivet PA, SantiagoBlay JA, Schmitt M (Eds) New development in the biology of Chrysomelidae. SPB Academic Publishing, The Hague, pp. 721-725.

Konstantinov AS, Korotyaev BA (2004) Sexual dimorphism and size of aedeagi in apionid weevils (Coleoptera: Apionidae) and flea beetles (Coleoptera: Chrysomelidae): Why some masculine males have small aedeagi? Proceedings of the Entomological Society of Washington 106: 324-338.

Lacordaire MT (1848) Monographie des Coléoptères subpentamères de la famille des Phytophages. Tome second. Bruxelles et Leipzig, 890 pp.

LeSage L (1982) The immature stages of Exema canadensis Pierce (Coleoptera: Chrysomelidae). Coleopterists Bulletin 36: 318-327.

LeSage L (1984) Immature stages of Canadian Neochlamisus Karren (Coleoptera: Chrysomelidae). Canadian Entomologist 116: 383-409.

Monrós F (1948) Descripción de diez nuevos Camptosoma neotropicales. Acta Zoologica Lilloana 6: 171-200.

Monrós F (1949) Descripción de seis nuevas Chlamisinae Neotropicales. (Col., Chrysomelidae). Revista de Entomología 20: 617-629.

Monrós F (1951) Notes on Chrysomelid beetles of the subfamily Chlamisinae, with descriptions of new species. Proceedings of the United States National Museum 101: 451-463.

Monrós F (1952) Revisión de las especies argentinas de Chlamisinae (Col., Chrysomelidae) (5a contribución al conocimiento de Chlamisinae). Acta Zoologica Lilloana 10: 489-672.

Navajas E (1944a) Algumas notas sobre a nomenclature dos Fulcidacideos (Col. Chrysomeloidea). Papeis avulsos do Departamento de Zoologia 4: 213-220.

Navajas E (1944b) Sobre a validez de Fulcidax violaceus (Klug, 1824) (Col. Fulcidacidae). Papeis avulsos do Departamento de Zoologia 4: 95-103.

Navajas E (1946) Os genotipos da Fam. Fulcidacidae (Col. Chrysomelidae). Papeis avulsos do Departomento de Zoologia 7: 243-245

Rafinesque CS (1815) Analyse de la nature ou tableau de l'univers et des corps organises. Palermo, $224 \mathrm{pp}$.

Reid CAM (1991) The Australian species of Chlamisini (Coleoptera: Chrysomelidae). Journal of the Australian Entomological Society 30: 315-323.

Riley EG, Clark SM, Flowers WR, Gilbert AJ (2002) 124. Chrysomelidae Latreille 1802. In: Arnett RH, Thomas MC, Skelley PE, Frank JH (Eds) American Beetles, Volume 2, CRC Press, Boca Raton, London, New York, Washington, D.C. 617-691. 
Riley EG, Clark SM, Seeno TN (2003) Catalog of the leaf beetles of America north of Mexico (Coleoptera: Megalopodidae, Orsodacnidae and Chrysomelidae, excluding Bruchinae). Coleopterists Society Special Publication No 1, Sacramento, 290 pp.

Seeno TN, Wilcox JA (1982) Leaf Beetle Genera (Coleoptera: Chrysomelidae). Entomography 1: $1-222$.

Voet JE (1806) Catalogus systematicus coleopterorum, vol. 2. La Haye, 82 pp. 